\title{
Monte Carlo calculation of backscattered light intensity by suspension: comparison with experimental data
}

\author{
Laurence Bergougnoux, Jacqueline Misguich-Ripault, \\ Jean-Luc Firpo, and Jacques André
}

\begin{abstract}
An optical device for measuring high particle concentrations is presented. The sensor consists of two optical fibers used for the emission and reception of the light scattered by particles in suspension. To describe the light path in this medium, we developed a Monte Carlo calculation including a mean free path, a phase function for particle scattering, and an absorption rate. A similarity law links concentration variations to a homothetic space transformation. A comparison between our calculation and experimental data is given for well-stirred, dense suspensions of silica particles with different sizes. A good fit is found when the model parameters (mean free path and phase function) are chosen according to the data given by a particle sizer.

Key words: Multiple scattering, Monte Carlo simulation, optical-fiber sensor, particle concentration. (c) 1996 Optical Society of America
\end{abstract}

\section{Introduction}

Turbidimetry measurements are widely used in biological investigations (Graaff et al. ${ }^{1}$ and references cited therein). Optical probes are also used for continuously measuring the particle concentration in liquid or gaseous suspensions ${ }^{2}$ (erosion, ${ }^{3,4}$ fluidized beds, slurry transport lines, sedimentation). Thus we designed ${ }^{4}$ such sensors made of optical fibers for emission and reception of the light intensity backscattered by particles in suspension in a transparent medium. As pointed out by Lischer and Louge, ${ }^{2}$ the main problem with this kind of sensor is in performing an accurate calibration of the voltage output in terms of the volume concentration of particles in suspension. Actually the light signal is a function not only of the concentration but also of numerous factors such as size, shape, complex refractive index of particles, and geometrical configuration of optical fibers. Our problem is in determining the

The authors are with the Institut Universitaire des Systemes Thermiques Industriels, Université de Provence, Unité Mixte de Recherche 139, Groupe d'Instrumentation des Procédeś et Systémes en Ecoulement, Turbidimétrie, Case 117, 13331 Marseille Cedex 3, France.

Received 26 April 1995; revised manuscript received 31 July 1995.

0003-6935/96/101735-07\$06.00/0

(1) 1996 Optical Society of America light intensity received by an optical-fiber core of diameter $D$ with acceptance cone $\beta$ at distance $d$ to another identical fiber that emits light (see Fig. 1).

This problem can be solved in the framework of the theory of radiative transfer. ${ }^{5}$ This phenomenological approach leads to the use of global parameters such as albedo and extinction length, which are not easy to interpret in terms of single-scatterer properties and particle concentration. The Green's-functions formalism ${ }^{6}$ gives some general properties of multiple scattering but, as a practical application, leads to unmanageable quadratures.

Thus Monte Carlo simulations, ${ }^{1,7}$ in spite of calculation time, seem well suited to performing calculations on backscattered light.

\section{Model}

To describe the suspension, we consider a collection of randomly distributed scatterers (see Fig. 2). So mean free path $\tilde{l}$ for a photon (a pencil of radiation in Chandrasekhar's terminology ${ }^{5}$ ) between two particles is given by

$$
\tilde{l}=\frac{1}{N \sigma_{\mathrm{sc}}} .
$$

In this expression $N$ is the number of scatterers per unit volume and $\sigma_{\mathrm{sc}}$ is the total scattering cross section of one particle. The probability density $P(l)$ 


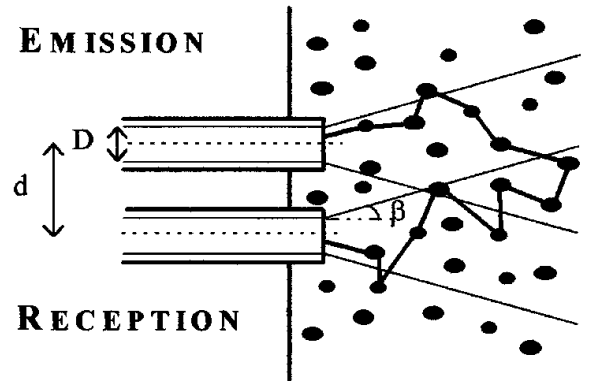

Fig. 1. Principle of the optical-fiber sensor based on backscattered light by particles in suspension.

that the photon goes over a distance $l$ is

$$
P(l)=\frac{1}{\tilde{l}} \exp \left(-\frac{l}{\tilde{l}}\right) .
$$

The suspension particles are characterized by

(1) A scattering law (phase function). Generally, the phase function is a function of $\mathbf{n}_{i}$ and $\mathbf{n}_{\text {sca }}$, the directions of incident and scattered radiations. If we consider spherical particles in suspension, the phase function depends only on $\cos \theta(\theta$ is the angle between $\mathbf{n}_{i}$ and $\mathbf{n}_{\text {sca }}$ ). We have two ways to choose this phase function.

(a) A phenomenological (functional) approach. For example, we choose the phase function given by Henyey-Greenstein. ${ }^{8}$ So the probability density for the scattering angle $\theta$ is

$$
P(\cos \theta)=\frac{1}{2} \frac{\left(1-g^{2}\right)}{\left(1+g^{2}-2 g \cos \theta\right)^{3 / 2}},
$$

where $g$ is a factor varying between -1 and 1 , which describes the anisotropy of an individual scatterer.

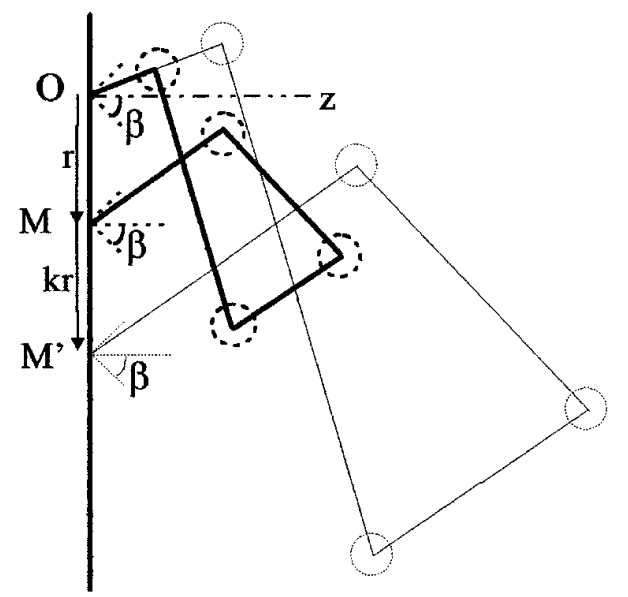

Fig. 2. Numerical simulation leads on this scheme. Photons are emitted at $\mathrm{O}$ in a random direction. After $n_{c}$ collisions, the photon reaches plane $z=0$ in $\mathrm{M}$ at distance $r$ to $\mathrm{O}$. After a homothetic transformation of center $\mathrm{O}$ and ratio $k$, the photon falls at point $\mathrm{M}^{\prime}$ at distance $k r$ to $\mathrm{O}$.
Physically we have $g=\langle\cos \theta\rangle$, the mean value of $\cos \theta$, and $\tilde{\theta}=\arccos (g)$. Thus forward scattering is assumed when $0<\tilde{\theta}<\pi / 2$ while backward scattering implies that $\pi / 2<\tilde{\theta}<\pi$. Isotropic scattering occurs when $\theta=\pi / 2$. In this case the $\cos \theta$ probability is constant.

(b) With the Mie theory and the size distribution function given by a particle sizer, we can determine a phase function for a mean particle. This method, initiated by Deirmendjian, ${ }^{9}$ is described in Section 6 . This phase function is called the Mie mean phase function.

(2) An absorption law. At every scattering event the probability of an incident photon being absorbed is $\tau$, so that the probability for an emergent photon after $n_{c}$ scatterings is

$$
P\left(n_{c}\right)=(1-\tau)^{n_{c}} .
$$

Our Monte Carlo calculation for multiple scattering is founded on these three probability laws [see Eqs. (2)-(4)]. The geometrical properties of the model are summarized in Fig. 2.

This problem is symmetrical with respect to the $z$ axis. So we determine $P_{\Delta r}(r)$, the sum of the contributions of photons falling down in the ring of radius $r$ and thickness $\Delta r$ around $\mathrm{O}$ in the plane $z=0$ :

$$
P_{\Delta r}(r)=\sum_{i=1}^{N_{\Delta r}(r)}(1-\tau)^{n_{c_{i}}}
$$

where $N_{\Delta r}(r)$ is the number of these photons and $n_{c_{i}}$ is the number of collisions experienced by the $i$ th photon. Now the light intensity received by the ring in arbitrary units is defined by

$$
I_{\Delta r}(r)=\frac{P_{\Delta r}(r)}{N_{\mathrm{ph}}}
$$

where $N_{\mathrm{ph}}$ is the total number of photons involved in a Monte Carlo experiment. This model is closely linked to the model described by Lischer and Louge ${ }^{2}$ and Groenhuis et al. ${ }^{7}$ Nevertheless the absorption is taken into account in a different way, and we emphasize the central part played by particle concentration [Eq. (1)].

\section{Similarity Law}

When a homothetic transformation of center $\mathrm{O}$ and ratio $k$ is performed on the photon path in Fig. 2, the photon reaching the $z=0$ plane in $\mathrm{M}$ at distance $r$ now falls into point $\mathrm{M}^{\prime}$ at distance $k r$ with the same number of collisions (same absorption) and the same scattering angles. Because light scattering by one particle is dependent only on the directions of the incident and emergent pencils of radiation, we deduce that

$$
I_{k \Delta r}(k r)=I_{\Delta r}(r)
$$


if the main free path $\tilde{l}$ of the photon becomes $k \tilde{l}$. In Eq. (1) the concentration in volume $c$ can be introduced and

$$
\tilde{l}=\frac{v}{c \sigma_{\mathrm{sc}}}=\frac{r_{0}}{c} .
$$

In this expression $v$ is the volume occupied by one particle and $r_{0}$ is the characteristic length of the suspension (see Section 6 for the case of polydisperse suspensions). We choose $r_{0}$ to be the unit length in our Monte Carlo simulation. Thus note that the geometric transformation $\tilde{l} \rightarrow k \tilde{l}$ is equivalent to changing the volume concentration $c \rightarrow c / k$, all other particle parameters remaining unchanged.

Hence, if $c$ is introduced into Eq. (7), we obtain

$$
I_{k \Delta r}\left(\frac{c}{k}, k r\right)=I_{\Delta r}(c, r)
$$

If $n$ is an integer, we deduce from Eq. (9)

$$
I_{\Delta r}(n c, r)=\sum_{i=0}^{n-1} I_{\Delta r}(c, n r+i \Delta r) .
$$

So, if $I_{\Delta r}(r)$ is known for values $r=0, \Delta r$, $\ldots, 1 \Delta r, \ldots$, at concentration $c_{0}$, we are able to calculate the corresponding quantities for the same value of $r$ at any concentration multiple of $c_{0}$.

This model describes the incoherent multiple scattering from the phase function of an isolated particle. This implies some restrictions in the density domain. At high densities the particles must be sufficiently distant (several wavelengths) in order that the emergent wave be spherical. We can estimate this limit at approximately $c \sim 0.1$ for our suspensions. Another limitation is given to this similarity law for the low densities because the photon path is bounded by the dimensions of the vessel that contains the suspension $\left(c \sim 10^{-4}\right.$ in our experiments). As we see in Section 7 the high concentration limitation is less drastic $(c \sim 0.3)$. This similarity law means physically that photons experience the same number of scattering events before they reach the observation plane. Only the distances of the impact point are dependent on the suspension volume concentration.

\section{Monte Carlo Process}

Calculation is performed for an arbitrary concentration $c_{0}$ (typically $c_{0}=0.001$ ) and $r_{0}=1$. In the following $\rho_{i}$ represents a random number uniformly distributed in the $[0,1]$ interval. This number is delivered by computer. The calculation is divided into four steps:

(1) Throwing of the photon in a random direction $(\theta, \phi)$ inside the acceptance cone $\beta$.

(2) Determination of the path length by

$$
l=-\tilde{l} \ln \left(1-\rho_{2}\right) .
$$

(3) Calculation of the new photon position.
(4) Choice of the photon emergent direction after collision with a particle. A local polar coordinate system is determined with the $z$ axis parallel to the incident photon direction. In this system we choose $\theta_{\mathrm{loc}}$ and $\phi_{\mathrm{loc}}$ for the emergent photon:

$$
\phi_{\text {loc }}=2 \pi \rho_{3}
$$

because we always consider spherical particles.

With the Henyey-Greenstein phase function, $\theta_{\text {loc }}$ is given by Groenhuis et al. ${ }^{7}$ :

$$
\theta_{\mathrm{loc}}=\arccos \left\{\frac{1}{2 g}\left[1+g^{2}-\frac{\left(1-g^{2}\right)^{2}}{\left(1-g+2 g \rho_{4}\right)^{2}}\right]\right\} .
$$

When we deal with a Mie mean phase function, $\theta_{\text {loc }}$ is determined numerically by the table lookup method. ${ }^{10}$

Now the direction of the emergent photon is calculated in the global coordinate system $(\theta, \phi)$, and we return to step $(2)$.

The process is stopped when the photon reaches plane $z=0$ or when the photon has experienced more than 10,000 collisions. If the photon is in the plane with a direction in acceptance cone $\beta$ of the receiving fiber, its distance $r$ to the origin and $n_{c}$ number of collisions are registered in a file. Now a new photon is launched until the total number of photons is achieved (typically more than 500,000 by file).

This file is characteristic of $\theta$. From this file, specifying $\tau$ the probability to be absorbed, $c$ the volume concentration, and $\Delta r$, the thickness of the ring of radius $\Delta r$, we build a set of $I_{\Delta r}(c, r)$ $(r=0, \Delta r, \ldots, n \Delta r, \ldots)_{\tilde{\sigma}}$ Figure $3(\mathrm{a})$ shows the results with forward $\left(\tilde{\theta}=27^{\circ}\right.$ and $\left.45^{\circ}\right)$, isotropic $\left(\tilde{\theta}=90^{\circ}\right)$, and backward $\left(\tilde{\theta}=135^{\circ}\right)$ scattering. Forward scattering in Eq. (13) spreads the photon distribution far from emitting point $O$. From this set of $I_{\Delta r}(c, r)$, results can be deduced for another concentration with Eq. (10). So our calculation gives us the light intensity in plane $z_{\tilde{\sigma}}=0$ at distance $r$ to the emitting point, specifying $\tilde{\theta}, \tau, c$, and $\Delta r$. In Fig. $3(\mathrm{~b})$ we represent for the same set of parameters the

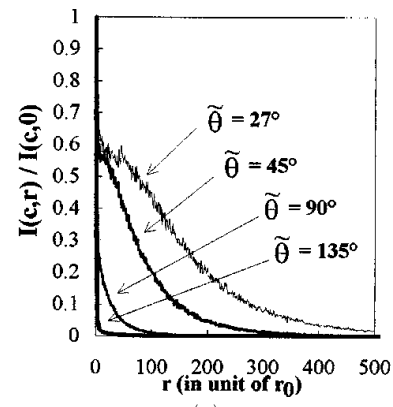

(a)

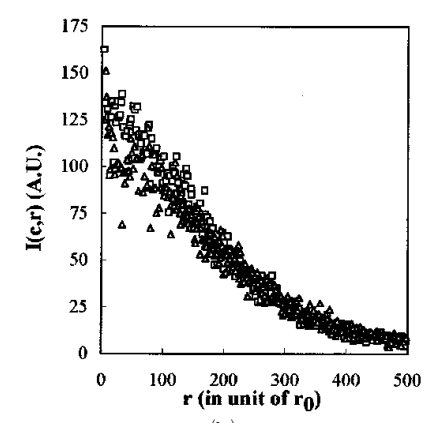

(b)
Fig. 3. Relative intensity on plane $z=0$ as a function of $r$ derived from the Monte Carlo process: (a) with several values of $\tilde{\theta}$ in the Henyey-Greenstein phase function, (b) with $(\square)$ the HenyeyGreenstein phase function and $(\triangle)$ a Mie mean phase function for the same anisotropy factor $\tilde{\theta}=27^{\circ}$. Results were obtained with $c=0.01, \tau=0.01$, and $\Delta r=10$. 
photon distribution versus distance for the HenyeyGreenstein phase function with $\tilde{\theta}=27^{\circ}$ and for a Mie mean phase function (see Section 6) with the same anisotropy factor $\langle\cos (\theta)\rangle=\cos \left(27^{\circ}\right)$. The distributions are very similar except near the origin.

A numerical integration on the section of the emitting fiber and on the receiving fiber (see Fig. 4) gives the calculated sensor response. So we can compare numerical results with experimental calibration curves and adjust $\tilde{\theta}$ and $\tau$ parameters. Consequently intensities deduced by quadratures by the Henyey-Greenstein or Mie mean phase function of the same anisotropy are nearly identical. In the following the Henyey-Greenstein formula is chosen.

\section{Experimental Procedure}

The method based on the light backscattering principle for determining the concentration ${ }^{2,11-14}$ or the particle size ${ }^{15}$ is now classical, but each device is different because of adjustment to the particle type and to the measurement range.

\section{A. Instrumentation and Measurement Techniques}

Figure 5 is a schematic of the calibration setup. The light from a He-Ne laser of $632.8-\mathrm{nm}$ wavelength is coupled at $1.6 \mathrm{~mW}$ onto a plastic optical fiber with a $D=750-\mu m$ core diameter, a 0.35 numerical aperture in water $\left(\beta=21^{\circ}\right)$, and a peak of

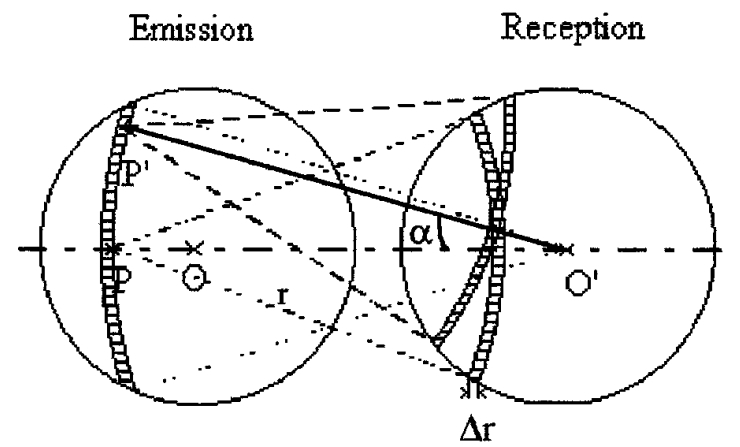

Fig. 4. Schematic diagram of the numerical integration on the section of the emitting and receiving fibers. For point $\mathrm{P}$ of the emitting fiber, photons arriving at distance $r$ in a slice of thickness $\Delta r$ on the receiving fiber are counted. Thus $r$ is varied to describe all the receiving fiber section. For the numerical integration on the emitting fiber a simple observation is made. Point $\mathrm{P}^{\prime}$ of the emission, obtained by rotation of center $\mathrm{O}^{\prime}$ and angle $\alpha$, gives the same number of photons with $(r, \Delta r)$ as with point $\mathrm{P}$.

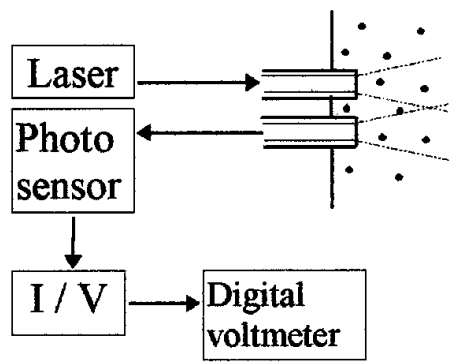

Fig. 5. Schematic diagram of the measuring system and the optical-fiber probe. transmittance at $580 \mathrm{~nm}$. Suspended particles reflect a fraction of the scattered light back to another identical fiber disposed at $d=760$ or $1520 \mu \mathrm{m}$ to the emitting fiber. The receiving light is conducted to a photodiode detector with a $0.4-\mathrm{A} / \mathrm{W}$ radiant sensitivity at $633 \mathrm{~nm}$. The photodiode short circuit current is converted in a dc potential. Thus we measure a voltage as a function of particles in suspension and an optical-fiber probe configuration. A detailed description of an experimental procedure is in Bergougnoux et al. ${ }^{4}$

\section{B. Particle Characteristics}

The response of our optical-fiber sensor is shown here for two silica types of particle [C800 and D066, Sifraco (France)]. Each particle-size variation is given by a Malvern MasterSizer E optical particle sizer. Both of these types of silica have, respectively, a $d_{32}=2.69-\mu \mathrm{m}$ (D066) and $d_{32}=1.06-\mu \mathrm{m}$ (C800) diameter volume/surface. The size distribution is shown in Figs. 6 and 7. Each silica, with a $2.65-\mathrm{g} \mathrm{cm}^{-3}$ density and a 1.544 real part of the index of refraction (relative to air), was suspended in water in a concentration range of $10^{-3}<c<0.3$. In this case the particle diameter is smaller than the core diameter of the optical fiber $\left(D / d_{32} \sim 279\right.$ or 714$)$. With this configuration, according to the conclusions of Lischer and Louge ${ }^{2}$ and Matsuno et al., ${ }^{13}$ the illuminated volume is sufficiently large to give an accurate determination of the mean concentration.

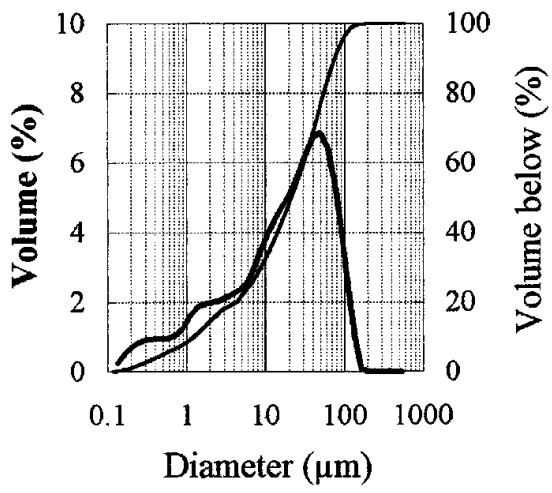

Fig. 6. D066 size distribution.

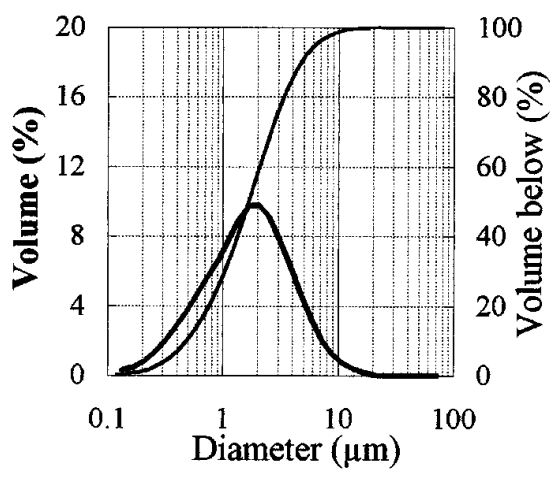

Fig. 7. C800 size distribution. 


\section{Results and Discussion}

\section{A. Experimental Results}

Calibration curves for each sample $(\boldsymbol{\Delta}, \boldsymbol{\nabla})$ show the backscattered light-intensity dependence on particle volume concentration (Figs. 8 and 9). With this device the intensity backscattered by particles and received by the optical fiber has a maximum, which depends on distance $d$ between the emitting and receiving fibers and on particle type (size, absorption, shape). For both samples a fiber set at $d=760 \mu \mathrm{m}$ receives more backscattering light and gives a calibration curve maximum abscissa $\sim 2.5$ times more than the same fiber set at $d=1520 \mu \mathrm{m}$. This effect is purely geometrical.

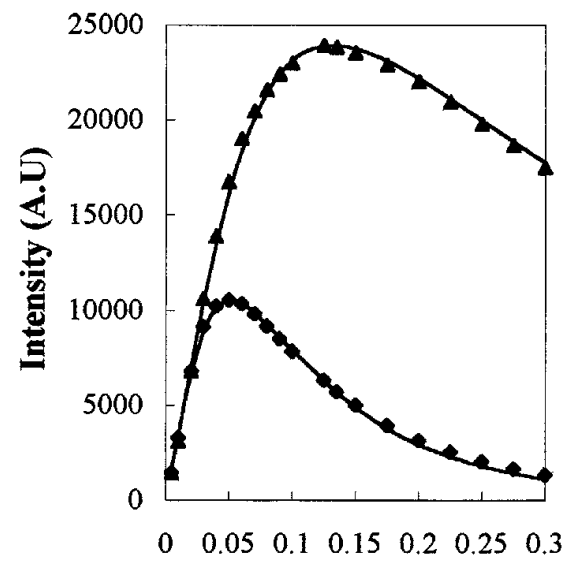

Volume concentration (-)

Fig. 8. D066. Comparison between $(\boldsymbol{\Lambda}, \diamond)$ experimental results and (-) numerical simulation as a function of volume concentration $c$. Experimental results are obtained with two fibers at $\boldsymbol{\Delta} d=760 \mu \mathrm{m}$ and $\bullet d=1520 \mu \mathrm{m}$ to the emission fiber. Numerical parameters are $\tau=2 \times 10^{-3}, r_{0}=3.6 \mu \mathrm{m}$, and $\tilde{\theta}=27^{\circ}$.

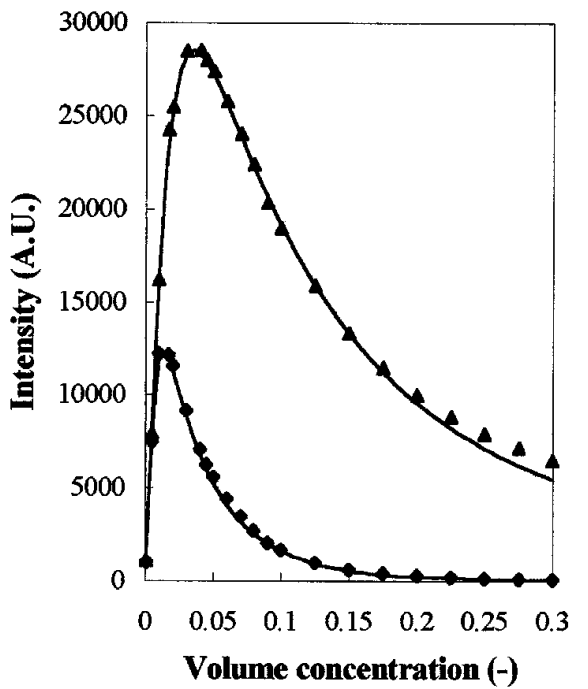

Fig. 9. C800. Comparison between $(\boldsymbol{\Lambda}, \diamond)$ experimental results and (-) numerical simulation as a function of volume concentration $c$. Experimental results obtained with two fibers at distance $\Delta d=760 \mu \mathrm{m}$ and $\boldsymbol{\Delta}=1520 \mu \mathrm{m}$ to the emission fiber. Numerical parameters are $\tau=5 \times 10^{-4}, r_{0}=1.08 \mu \mathrm{m}$, and $\tilde{\theta}=30^{\circ}$.
C800 suspension attenuates light of less than D066 suspension. We recognize the classical result of scattering theory. ${ }^{9,16,17}$ The curve maximum abscissa increases with the size of the particles in suspension. During this test we noted good reproducibility made easier by suspension homogeneity. Thus we can estimate a concentration measurement precision $^{4}$ of $\pm 2 \%$.

To fit our experimental data by the Monte Carlo simulation (see Section 4), we must adjust some parameters: the suspension characteristic length $r_{0}$, the scattering phase function, the probability $\tau$ of a photon being absorbed during the collision.

\section{B. Choice of $r_{0}$}

For polydisperse suspensions the particle sizer gives the distribution of particles in several size classes ( $N_{c l}$, the total number of classes). We have $n_{i}$ particles in the class $i$ per unit volume of suspension. Each particle of this class has a volume $v_{i}=(4 / 3) \pi \rho_{i}{ }^{3}$ if it is supposed spherical with radius $\rho_{i}$. If $c$ is the volume concentration of suspension, we have for the unit volume

$$
\sum_{1=1}^{N_{c l}} n_{i} v_{i}=c
$$

For a spherical particle with radius $\rho_{i}$, the Mie theory ${ }^{16,17}$ gives for a $\sigma_{\mathrm{sc}, i}$ scattering cross section of class $i$ :

$$
\sigma_{\mathrm{sc}, i}=\frac{2}{x_{i}{ }^{2}} \sum_{n=1}^{\infty}(2 n+1)\left\{\left|a_{n}\right|^{2}+\left|b_{n}\right|^{2}\right\} \pi \rho_{i}{ }^{2} .
$$

In this expression $x_{i}=\left(2 \pi \rho_{i}\right) / \lambda, a_{n}$ and $b_{n}$ are the Mie coefficients, and $\lambda$ is the wavelength of laser light in the medium (water).

If we note $p_{i}$, the relative volume probability for class $i$, we have for the unit volume of suspension

$$
n_{i} v_{i}=p_{i} c .
$$

So, according to Eq. (8), the mean free path $\tilde{l}_{i}$ for the $i$ th class is

$$
\tilde{l}_{i}=\frac{v_{i}}{c p_{i} \sigma_{\mathrm{sc}, i}} .
$$

Thus we have $N_{c l}$ mean free paths. With Eq. (11), $N_{c l}$ photon path lengths can be generated. The right path is the minimum path because the simulated photon is scattered by the first particle found:

$$
l=\min \left(l_{1}, l_{2}, \ldots, l_{N_{c l}}\right) .
$$

The random variable $l$ follows an exponential probability law with a mean free path $\tilde{l}$ :

$$
\tilde{l}=\frac{1}{\sum_{i=1}^{N_{c l}} \frac{1}{\tilde{l}_{i}}} .
$$

1 April 1996 / Vol. 35, No. 10 / APPLIED OPTICS 
Consequently $r_{0}$ of Eq. (8) is given by

$$
r_{0}=\frac{1}{\sum_{i=1}^{N_{c l}} \frac{p_{i} \sigma_{s c, i}}{v_{i}}} .
$$

C. Determination of the Phase Function

In Eq. (16) the probability that $l$ belongs to class $i$ is given by

$$
q_{i}=\frac{\frac{1}{\tilde{l}_{i}}}{\sum_{j=1}^{N_{c l}} \frac{1}{\tilde{l}_{j}}}=r_{0} \frac{p_{i} \sigma_{s c, i}}{v_{i}} .
$$

With our problem $q_{i}$ is the probability in order that the photon be scattered by a particle of class $i$. With the Mie theory we know the phase function for a spherical particle $\phi\left(\rho_{i}, m, \theta\right)$, where $m$ is the complex refractive index of suspension particles. So the Mie mean phase function $\phi_{M m}$ is given by

$$
\phi_{M m}(m, \theta)=\sum_{i=1}^{N_{c l}} q_{i} \phi\left(\rho_{i}, m, \theta\right) .
$$

Parameter $g$ of the Henyey-Greenstein formula is given by $\langle\cos \theta\rangle$ calculated with $\phi_{M m}(m, \theta)$.

\section{Absorption Rate Choice}

The implicit assumption made by optical particle sizers is that particles are spherical. In other cases, if we want a good size analysis and consequently well-fitted phase functions, we must adjust the imaginary part of the refractive index to obtain the concentration calculated by the software device equal to the sample concentration. So doing, for nonspherical particles such as our silica, we overestimate the imaginary part of the refractive index, and the calculations of $K_{\mathrm{abs}, i}$ quantities in the framework of Mie theory are unrealistic. The silica suspension should be black! Thus we adjust $\tau$ for a best fit with the experimental curves. Note that $\tau$ is smaller for C800 silica than for D066 silica. This fact is not surprising because C800 is smaller than D066.

The choice of parameters for both silica suspen-

\begin{tabular}{|c|c|c|c|c|c|}
\hline Silica Type & $\begin{array}{c}\text { Diameter } \\
\text { Volume/ } \\
\text { Surface } d_{32} \\
(\mu \mathrm{m})\end{array}$ & $\begin{array}{c}r_{0} \\
(\mu \mathrm{m})\end{array}$ & $\begin{array}{c}\tilde{\theta} \\
(\operatorname{deg})\end{array}$ & $g=\langle\cos \theta\rangle$ & $\tau$ \\
\hline D066 & 2.69 & 3.6 & 27 & 0.891 & $2 \times 10^{-3}$ \\
\hline C800 & 1.06 & 1.08 & 30 & 0.866 & $5 \times 10^{-4}$ \\
\hline
\end{tabular}
sions is summarized in Table 1.

Table 1. Parameters for Silica Suspensions

\section{Conclusion}

Figures 8 and 9 show good agreement between the experimental results and those of the numerical simulation with the parameters mentioned above, obtained for both silica samples.

The model is well suited for describing the sensor response curves in a large range of concentration. Particularly, in the high-concentration domain $c>$ 0.1 , it gives a better fit than expected. We think that this right fit is due more to the application of the similarity law in the calculations than to the choice of the scattering particle parameters $\left(r_{0}, \tilde{\theta}, \tau\right)$. In fact several choices of these parameters can lead to the same right fit with experimental concentration curves. In Section 6 we gave a method for deducing $r_{0}$ and $\tilde{\theta}$ from the data delivered by a particle sizer. For the present the value of $\tau$ is adjusted to fit experimental values; to check it we are designing an integrating sphere device ${ }^{18}$ for direct measurement of $\tau$.

The use of the Henyey-Greenstein formula to describe the scattering properties of an individual particle leads to the same results that the use of a Mie mean function with the same anisotropy does. Other approaches should give the mean scattering phase function for various particle shapes..$^{19,20}$

We are grateful to R. Blanc (Laboratoire de Physique des Systèmes désordonnés, Université de Provence) for many valuable discussions and the use of the Malvern optical particle sizer. This research was supported by the Cemagref and the Conseil Régional Provence Alpes Côte d'Azur. It is part of the Ph.D. dissertation of L. Bergougnoux sponsored by the Ministère de l'Enseignement Supérieur et de la Recherche. The authors are with Université de Provence, Institut Universitaire des Systemes Thermiques Industriels, Unité Mixte de Recherche 139, Groupe d'Instrumentation des Procédés et Systémes en Encoulement-Turbidimétrie, case 117, 13331 Marseille Cedex 3, France.

\section{References}

1. R. Graaff, M. H. Koelink, F. F. M. de Mul, W. G. Zijlstra, A. C. M. Dassel, and J. G. Aarnoudse, "Condensed Monte Carlo simulations for the description of light transport," Appl. Opt. 32, 426-434 (1993).

2. D. J. Lischer and M. Y. Louge, "Optical fiber measurements of particle concentration in dense suspensions: calibration and simulation," Appl. Opt. 31, 5106-5113 (1992).

3. C. S. Conner and A. M. De Visser, "A laboratory investigation of particle size effects on an optical backscatterance sensor," Mar. Geol. 108, 151-159 (1992).

4. L. Bergougnoux, J. Misguich-Ripault, J-L. Firpo, J. André, J-P. Cambon, N. Mathys, and J-E. Olivier, "Mesure in situ de la concentration des matières en suspension: mise au point d'une sonde optique," in Compte-rendu de recherche 3, BVRE de Draix (Cemagref ed., Grenoble, 1995), pp. 63-77.

5. S. Chandrasekhar, Radiative Transfer (Dover, New York, 1960), Chap. 1, p. 1.

6. S. M. Rytov, Yu. A. Kravtsov, and V. I. Tatarskii, Principles of Statistical Radiophysics (Springer-Verlag, Berlin, 1989), Vol. 4, Chap. 4, p. 117. 
7. R. A. J. Groenhuis, H. A. Ferwerda, and J. J. Ten Bosch, "Scattering and absorption of turbid materials determined from reflection measurements. I: Theory," Appl. Opt. 22, 2456-2462 (1983).

8. H. C. van de Hulst, Multiple Light Scattering (Academic, New York, 1980), Vol. 2, Chap. 11, p. 331.

9. D. Deirmendjian, Electromagnetic Scattering on Spherical Polydispersions (Elsevier, New York, 1969), pp. 72-93.

10. I. Lux and L. Koblinger, Monte Carlo Particle Transport Methods: Neutrons and Photons Calculations (CRC Press, Boca Raton, Fla., 1991), pp. 12-13.

11. H. Yamazaki, K. Tojo, and K. Miyanami, "Measurement of local solids concentration in a suspension by an optical method," Powder Technol. 70, 93-96 (1992).

12. G. C. Kineke and R. W. Sternberg, "Measurements of high concentration suspended sediments using the optical backscatterance sensor," Mar. Geol. 108, 253-258 (1992).

13. Y. Matsuno, H. Yamaguchi, T. Oka, H. Kage, and K. Higashitani, "The use of optic fiber probes for the measurement of dilute particle concentrations: Calibration and application to gas-fluidized bed carryover," Powder Technol. 36, 215-221 (1983).

14. A. S. Bos and J. J. Heerens, "Light backscattering as a technique to measure solid particle size and concentration in suspension," Chem. Eng. Commun. 16, 301-311 (1982).

15. H. S. Dhadwal, R. R. Ansari, and W. V. Meyer, "A fiber-optic probe for sizing in concentrated suspensions," Rev. Sci. Instrum. 62, 2963-2968 (1991).

16. C. F. Bohren and D. R. Huffman, Absorption and Scattering of Light by Small Particles (Wiley, New York, 1983), pp. 69-73.

17. H. C. van de Hulst, Light Scattering by Small Particles (Wiley, New York, 1957), Chap. 9, p. 114.

18. F. Desvignes, Rayonnements Optiques Radiométrie Photométrie (Masson, Paris, 1991), pp. 211-213.

19. M. I. Mishchenko and L. D. Travis, "Light scattering by polydispersions of randomly oriented spheroids with sizes comparable to wavelengths of observations," Appl. Opt. 33, 7206-7225 (1994).

20. E. H. Hirst, P. H. Kaye, and J. R. Guppy, "Light scattering from nonspherical airborne particles: experimental and theoretical comparisons,"Appl. Opt. 33, 7180-7186 (1994). 